\title{
Procesos cognitivos y rendimiento en la acción de saque en voleibol
}

\section{Manuel CONEJERO ${ }^{1}$, Fernando CLAVER ${ }^{1}$, Carmen FERNÁNDEZ-ECHEVERRÍA ${ }^{1}$, Jara GONZÁLEZ-SILVA ${ }^{1}$ y M. Perla MORENO ${ }^{1}$}

${ }^{1}$ Universidad de Extremadura. España

(Recibido, 11 marzo 2017; Aceptado, 18 mayo 2017)

\begin{abstract}
RESUMEN: El saque es una de las acciones de juego en voleibol a la que los equipos han dado gran importancia, definiéndose como la acción de poner el balón en juego por el jugador zaguero derecho, situado en la zona de saque. Ésta es la única acción en la que el jugador tiene el 100\% del control de la pelota, teniendo tiempo suficiente para elegir el tipo, la fuerza y el área donde irá dirigido. Además, es considerado acción finalista ya que se puede conseguir punto a través de él, pudiendo afectar al rendimiento del equipo. El objetivo de este estudio fue analizar la relación entre la toma de decisiones y el rendimiento en la acción de saque, en voleibol de categoría Juvenil Masculino. Se analizaron mediante la observación sistemática 1555 acciones de saque, correspondientes a los 21 equipos participantes en el Campeonato de España Juvenil Masculino, celebrado en 2012. Los resultados mostraron una asociación significativa entre la toma de decisiones y el rendimiento, en la acción de saque $\left(.337^{* *}\right)$. Además, existe una asociación alta entre ambas variables (Gamma $=.533$ ), y un nivel de dependencia moderado entre las mismas $(\mathrm{D}$ de Somers $=.315)$. Estos resultados muestran la importancia de poseer adecuadas capacidades decisionales para obtener un óptimo rendimiento en la acción de saque, y contribuir así a mejorar el rendimiento del equipo. Por tanto, en el proceso de entrenamiento de jugadores de categoría juvenil sería apropiado considerar la posibilidad de empleo de actividades que contribuyan a la mejora de la capacidad decisional de los deportistas.
\end{abstract}

Palabras clave: Voleibol, toma de decisiones, rendimiento, saque.

\section{Cognitive processes and performance in the serve action in volleyball}

\begin{abstract}
The serve is one of the game actions in volleyball to which teams have given great importance. It is defined as the action to start the rally by the right back player, located in the serve zone. The serve is a single action in which the player has $100 \%$ control of the ball, having enough time to choose the type, strength and area where it will be directed. In addition, it is considered a finalist action as a direct point can be achieved through it, affecting the team performance. The objective of this study was to analyze the relationship between decision-making and performance in the serve action, in volleyball U19 category. 1555 serve actions were analyzed through the systematic observation of the 21 teams participating in the U-19 Male Spanish Championship in 2012. The results showed a significant association between decision-making and performance in the serve action $\left(.337^{* *}\right)$. In addition, there is a high association between both variables (Gamma $=.533)$, and a moderate dependency level between them (Somers' $\mathrm{D}=.315$ ). These results show the importance of having adequate decision-making abilities to obtain optimum performance in the serve action, improving team performance. Therefore, in the
\end{abstract}


training process of youth volleyball players it would be appropriate to use activities to improve the players' decision-making abilities.

Keywords: Volleyball, decision- making, performance, serve.

Correspondencia: Manuel Conejero Suárez. Avd. de la Universidad s/n. Facultad de Ciencias del Deporte. 10003 Cáceres. Tfno: 616849 008. Email: mconejerx@alumnos.unex.es

\section{Introducción}

En el estudio de la pericia en el deporte, y centrándonos en el proceso de toma de decisiones, podemos encontrar distintas aproximaciones. Tradicionalmente se han utilizado diferentes perspectivas y metodologías para su estudio, destacando la perspectiva de la psicología cognitiva y la perspectiva ecológica (Bar-Eli \& Raab, 2006). Aunque son dos marcos conceptuales distintos, ambos tienen el objetivo de estudiar y comprender la toma de decisiones para la mejora del rendimiento.

El enfoque de la perspectiva ecológica propone que la información más relevante para la toma de decisiones y el aprendizaje, en entornos dinámicos, surge de las interacciones continuas entre el sujeto y el ambiente, dando una gran importancia a los procesos de percepción (Araújo, Davids \& Hristovski, 2006; Van Orden, Holden \& Turvey, 2003). Por su parte, desde la perspectiva cognitiva, el rendimiento de los deportistas depende de las representaciones mentales internas y de los procesos cognitivos que median entre la interpretación de un estímulo y la selección de la respuesta (Hodges, Starkes \& MacMahon, 2006; Sutton \& Mcilwain, 2015).

Existen dos modelos para el estudio de la toma de decisiones que han sido utilizados dentro de la perspectiva cognitiva: un primer modelo, centrado con el estudio de los parámetros temporales y visuales; y un segundo modelo, orientado al estudio de los procesos relacionados con la memoria (Bar-Eli \& Raab, 2006).

En el estudio de la toma de decisiones, dentro del modelo de los parámetros temporales y visuales, se analiza la percepción del deportista antes de la acción, donde se tiene en cuenta el tiempo de reacción (Sternberg, 1969). Según este modelo, se considera que la información que el deportista recibe de manera visual puede servir para disponer de un mayor tiempo para seleccionar la respuesta y así poder anticiparse. Esta información que es recibida de manera visual puede ser utilizada para la selección de la respuesta exitosa (Williams, Ward, Smeeton \& Allen, 2004).

En el modelo orientado al estudio de los procesos relacionados con la memoria, se considera que las estructuras de conocimiento almacenadas en la memoria condicionan la toma de decisiones, por lo que cuanto mayor y más variado sea el conocimiento del deportista, sobre el deporte, mejor será la toma de decisiones (Köppen \& Raab, 2009). Por tanto, el conocimiento del deportista sobre el deporte que práctica, servirá de base para una selección efectiva de la respuesta (Starkes, Helsen \& Jack, 2001; Williams, Davids \& Williams, 1999).

La toma de decisiones puede ser definida como el proceso por el cual el deportista elige cómo actuar o reaccionar a las demandas del entorno para conseguir objetivos de rendimiento (Hodges, Huys \& Starkes, 2007). En este sentido, supone la detección de una información adecuada del entorno para planificar acciones futuras, y poder así, hacer frente a las contingencias del juego (Baker, Whiting \& Van der Brugg, 1992). Por lo que, podemos considerar la toma de decisiones, en una situación deportiva, como la selección de la respuesta 
más adecuada en las distintas condiciones de juego (Conejero, Claver, Fernández-Echeverría, Gil-Arias \& Moreno, 2017).

Por su parte, Ruiz, Sánchez y Piqueras (2006) determinaron que en los deportes de carácter abierto, es difícil que ocurran las mismas situaciones una y otra vez, y hay que intentar que el oponente conozca lo menos posible como actuaremos. Cuanto más abierta sea la habilidad deportiva, mayor número de estímulos para percibir y procesar, por lo que la acción será aún mas compleja en términos de toma de decisiones (Gregháine et al., 2001). Así por ejemplo, en voleibol hay un amplio campo de incertidumbre, por lo que la selección de respuesta será compleja en los diferentes momentos del juego (Thomas \& Thomas, 1994). Por ello, y debido a las características particulares que se dan en el contexto deportivo, y concretamente en los deportes colectivos, el estudio de la toma de decisiones (Johnson, 2006) y de los procesos cognitivos (Moran, 2012), en los mismos es fundamental.

Los factores cognitivos son uno de los dominios que afectan al rendimiento, unido a los dominios fisiológico, emocional y técnico (Janelle \& Hillman, 2003). Dependiendo de las características propias de cada deporte estos dominios van a influir en mayor en menor medida. En aquellos deportes de carácter abierto donde hay gran incertidumbre de estímulos, los procesos cognitivos y el dominio técnico serán dos de los factores más importantes en el rendimiento (Starkes, 1993).

Numerosas investigaciones han tratado de analizar las relaciones existentes entre conocimiento, toma de decisiones y rendimiento en el deporte (Hastie, Sinelkinov \& Guarino; Iglesias, Moreno, Santos-Rosa, Cervelló \& Del Villar, 2005; Nielsen \& McPherson, 2001). Los resultados de estos esutidos ponen de manifiesto la existencia de una correlación positiva y significativa entre las variables cognitivas y el rendimiento (French \& Thomas, 1987; Iglesias, Sanz, García-Calvo, Cervelló \& Del Villar, 2005; McPherson, 1993).

Concretamente, en voleibol, Moreno et al. (2006) y Murray (1991), analizaron la relación entre el conocimiento, aspectos decisionales, aspectos de ejecución y rendimiento del equipo. En ambos estudios los resultados determinaron que una mayor experiencia y conocimiento del voleibol, permitió al deportista una mejor toma de decisiones y, por lo tanto, mayor rendimiento en las diferentes acciones de juego, lo que llevó a los equipos que tenían jugadores con mejor toma de decisiones a ocupar mejores puestos en la clasificación final de la competición.

En esta investigación se analizará la relación existente entre la toma de decisiones y el rendimiento en la acción de saque en voleibol. En voleibol las acciones que se realizan son cíclicas y secuenciales (Beal, 1989; Fraser, 1988) produciéndose en tres complejos de juego fundamentales K0, K1 y K2 (Monge, 2007). El complejo 0 (K0) inlcuye la acción de saque, mediante la cual se pone en juego el balón. El complejo 1 (K1) es conocido como la fase de ataque e incluye las acciones de recepción, colocación, ataque y cobertura al ataque (Selinger \& Ackermann-Blount, 1985). El objetivo principal de este complejo de juego es recibir óptimamente el saque para realizar la organización ofensiva mediante un buen ataque, y así lograr el punto y la posesión del saque (Papadimitriou, Pashali, Sermaki, Mellas \& Papas, 2004). El complejo 2 (K2) es conocido como la fase de defensa e incluye las acciones de bloqueo, defensa en campo, colocación, contraataque y cobertura al contraataque (Rodriguez-Ruiz et al., 2011). El objetivo principal del K2 es neutralizar y contrarrestar el ataque del equipo contrario, posibilitando una optima construcción de contraataque, que permita la consecución del punto y la continuidad en la posesión del saque (Ureña, Calvo \& Lozano, 2002). 
El saque es una de las acciones a la que los equipos han dado gran importancia, definiéndose como la acción de poner el balón en juego por el jugador zaguero derecho, situado en la zona de saque (REFVB, 2013:41). Debido a la posibilidad de conseguir un punto a través del saque, éste se considera una acción finalista, pudiendo afectar al rendimiento del equipo (Drikos, Kountouris, Laios \& Laios, 2009). A nivel decisional, el saque es la única acción de juego en la que el jugador tiene el $100 \%$ del control sobre la pelota, teniendo suficiente tiempo para elegir el tipo de servicio, la fuerza que se imprime a la pelota y el área a la que el servicio estará dirigido (Ureña, Santos, Martínez, Calvo \& Oña, 2010).

Pocos son los estudios que han tratado de analizar la relación entre la toma de decisiones y el rendimiento en una acción de características tan particulares como la acción de saque en voleibol. Por ello, el objetivo fundamental de la presente investigación es analizar la correlación, asociación y dependencia existente entre la toma de decisiones y el rendimiento en la acción de saque, en voleibol de categoría Juvenil Masculino.

\section{Método}

\section{Participantes}

La muestra de estudio estuvo compuesta por 1555 acciones de saque realizadas por los 221 jugadores, del total de los 21 equipos participantes del Campeonato de España de clubes de categoría Juvenil Masculino, celebrado en 2012.

Se realizó la observación sistematica de las acciones de juego en dos partidos de cada uno de los equipos particpantes en el campeonato. Todos los partidos correspondían a la primera fase de de dicho campeonato.

\section{Variables e Instrumentos}

Las variables de estudio consideradas en la investigación son de dos tipos: cognitivas (toma de decisiones) y de rendimiento (rendimiento en juego).

- La toma de decisiones en la acción de saque, definida como el proceso a través del cual un deportista selecciona una acción entre una serie de alternativas, para ejecutarla en una situación real de juego (Tenembaum, 2004), teniendo en cuenta las demandas del entorno, para así poder lograr los distintos objetivos de rendimiento (Hodges et al., 2007). La toma de decisiones fue medida a través del Game Performance Assesment Instrument (GPAI), elaborado por Oslin et al. (1998). Este instrumento es utilizado para observar y codificar acciones deportivas que demuestran la habilidad del jugador en la resolución de situaciones tácticas. Con dicho instrumento se evalúan 7 factores, analizándose en el presente estudio únicamente el referente a la toma de decisiones. Para cada acción se asignaba un valor 1 a aquellas decisiones consideradas apropiadas, que cumplía alguno de los criterios establecidos, y un valor 0 para aquellas inapropiadas, que no cumplían dichos criterios. El instrumento empleado para la valoración de la toma de decisiones en la acción de saque en voleibol fue validado y empleado en estudios previos (Moreno, Moreno, García-González, Gil, Claver \& Del Villar, 2011). 
- El rendimiento en la acción de saque, entendido como el resultado final de la ejecución motriz. La medida se realizó a través del Sistema de observación de la Federación Internacional de Voleibol, FIVB (adaptado de Coleman, 1975). Dicho instrumento consta de una escala en la que se otorgan valores de 0 a 4 en la acción de saque en voleibol. Donde 0 supone punto para el oponente (falta o saque a varilla, red o fuera), 1 es el saque que permite todas las opciones de ataque tras ser recibido, 2 es el saque que limita las opciones de ataque tras la recepción, no permitiendo tiempos rápidos de ataque, 3 es el saque que provoca un free o balón que pasa directamente al campo contrario tras ser recibido, y 4 es el saque que permite la ejecución exitosa de la acción ya sea por punto directo o fallo en recepción como consecuencia del saque.

\section{Procedimiento}

Para la observación sistematica de la toma de decisiones y el rendimiento en la acción de saque se registraron grabaciones de los diferentes partidos. Las grabaciones se realizaron con una cámara digital SONY HDR-XR155 sobre formato M2TS. La cámara se ubicó en uno de los fondos de la cancha de juego a una altura de 5 metros sobre el suelo, lo cual permitió obtener un plano similar en las diferentes grabaciones.

Una vez realizada la recogida de datos se procedió a la observación de todos los partidos por un mismo observador. Para garantizar la fiabilidad de la observación, un observador con experiencia en esta función (Graduado en Ciencias de la Actividad Física y el Deporte, Entrenador Nacional Nivel III de voleibol, y con experiencia como entrenador), realizó un proceso de entrenamiento en el que se utilizaron, en las diferentes sesiones de entrenamiento, muestras con distintas características (partidos de equipos de distinta posición en la clasificación final del campeonato), y superando el $10 \%$ de la muestra total, indicado por Tabachnick \& Fidell (2007). Se alcanzaron en la observación de todas las variables unos valores de Kappa de Cohen intra observador superiores a .75 (Fleiss, Levin \& Paik, 2003).

\section{Análisis estadístico}

Se utilizó el programa estadístico SPSS 21.0 como apoyo informático para el análisis de los datos recogidos. Se realizó un análisis de correlación, y además un análisis inferencial para comprobar el nivel de asociación y el grado de dependencia de la acción de saque en sus diferentes variables (toma de decisiones y rendimiento). Este análisis se presenta a través de los valores de Rho Spearman, el coeficiente de Gamma de Goodman y Kruskal, y el coeficiente D de Somers, teniendo en cuenta que el nivel de significatividad fue de $\mathrm{p}<.05$.

\section{Resultados}

\section{Análisis de correlación entre la toma de decisiones y el rendimiento en la acción de saque}

El análisis estadístico nos muestra que existe una asociación significativa entre la toma de decisiones y el rendimiento en la acción de saque (Tabla 1). Además, existe una asociación 
alta entre ambas variables $(\mathrm{Gamma}=.533)$, y un nivel de dependencia moderado entre las mismas (D de Somers $=.315$ ).

Tabla 1. Correlaciones bivariadas del saque.

\begin{tabular}{lcc}
\hline Variables & $\mathbf{1}$ & $\mathbf{2}$ \\
\hline 1.Toma decisiones saque & - & \\
2.Rendimiento saque & $.337^{* *}$ & - \\
\hline$*$ & La correlación es significativa en el nivel 0.05 & \\
$* *$ & La correlación es significativa en el nivel 0.01 &
\end{tabular}

\section{Discusión}

El objetivo principal de la investigación fue analizar la correlación, asociación y dependecia existente entre la toma de decisiones y el rendimiento en la acción de saque, en voleibol de categoría Juvenil Masculino.

Estudios en diferentes deportes han mostrado relaciones significativas entre la toma de decisiones y el rendimiento en juego (McPherson, 1999; Nielsen \& McPherson, 2001). De forma concreta, en beisbol, los jugadores que tomaban decisiones acertadas mostraron un mayor rendimiento (McPherson, 1993), ocurriendo lo mismo en deportes como el bádminton (Hastie el al., 2009), y el tenis (García-González, Moreno, Moreno, Iglesias, \& Del Villar, 2009). Por tanto, el desarrollo de decisiones tácticamente adecuadas por parte de los jugadores expertos, unido a la realización de óptimas ejecuciones, determina el éxito conseguido en las diferentes acciones de juego (A. Moreno et al., 2011).

En nuestro estudio, la toma de decisiones en la acción de saque correlaciona significativamente con el rendimiento del saque, existiendo una asociación alta entre ambas variables y un nivel de dependencia moderado entre las mismas. Resultados similares se obtuvieron en el estudio desarrollado por M. P. Moreno et al. (2011), en voleibol en la acción de saque con una muestra de la Selección Nacional Absoluta Femenina, donde la toma de decisiones de las jugadoras en la acción de saque correlacionó con el rendimiento en dicha acción.

El saque es la segunda acción de juego, después del ataque, que mayor correlación tiene con la consecución del punto (Fernades \& Moutinho, 1996). Unido a ello, el rendimiento del saque también se manifiesta en función de su capacidad de obstaculizar el ataque después de la recepción (Ureña, Santos, Martínez, Calvo \& Oña, 2010). El saque en voleibol posee unas características particulares, siendo la única acción de juego en la que el jugador tiene el $100 \%$ del control sobre la pelota y sobre el desarrollo los acontecimientos, debiendo elegir el jugador el tipo de saque, la fuerza que le imprimirá a la pelota y el área a la que el saque irá dirigido (Fernández-Echeverría, Claver, Gil, Del Villar \& Moreno, 2015). Por tanto, una buena decisión, en relación a dichas variables, posibilitarán un mejor rendimiento del jugador, ya que, de acuerdo con nuestros resultados existe dependencia entre la toma de decisiones y el rendimiento.

Según la escala de dificultad creada por Ureña y González (2006), el saque tiene una dificultad baja en lo que a toma de decisiones se refiere, debido a que el tiempo requerido para tomar la decision es amplio (1-8 segundos), además el nivel de riesgo del objetivo es nulo, y los elementos a recordar son varios, sin gran confusión de estímulos. Por su parte, en cuanto a la dificultad técnica del saque se puede decir que es una habilidad sencilla ya que el tamaño de la (c) Psy, Soc, \& Educ, 2017, Vol. 9(3) 
diana es grande y se dispone de tiempo para decidir, observar y elegir el tipo de saque que mejor ejecuta el jugador. El saque es la tarea motriz del voleibol con menores exigencias con relación a los tres mecanismos comportamentales (percepción, decisión y ejecución), y también la que menor presión temporal presenta en su realización (Ureña \& González, 2006). Debido a este menor nivel de dificultad de la acción de saque, en relación con el resto de acciones de juego que acontecen en voleibol, un óptimo proceso de entrenamiento técnico y táctico de la acción de saque podría contribuir en gran medida a mejorar el rendimiento de dicha acción.

De acuerdo con ello, los jugadores de voleibol, en función de su nivel de dominio del saque, asumirán diferente riesgo en distintos momentos (Palao, Santos \& Ureña, 2004), optarán por utilizar diferentes tipos de saque, o sacarán partido a determinadas variables manifestadas como determinantes o predictoras de la eficacia del saque en estudios precedentes (Afonso, Esteves, Araújo, Thomas \& Mesquita, 2012; Ureña, Vavassori, León \& González, 2011). En este sentido, en categorías de formación en voleibol (infantil y cadete) las variables zona de origen del saque, tipo de saque, zona de recepción y función del receptor, se han manifestado como variables predictoras de la eficacia del saque (Fernández-Echeverría, Gil, Moreno, Claver \& Moreno, 2015).

Los resultados de nuestro estudio muestran que la toma de decisiones en la acción de saque, se asocia al rendimiento en dicha acción en voleibol de categoría Juvenil Masculina. En el deporte, rendir consiste en elegir la opción de juego más adeucada en cada momento y ser capaz de llevarla a cabo de forma eficiente durante todo el partido (Gréhaigne et al., 2001). De esta forma, se puede asumir que tomar mejores decisiones puede suponer una mejora del rendimiento (Hepler \& Chase, 2008), poniéndose de manifiesto la posible relación lineal entre ambas variables (Hastie et al., 2009; Iglesias et al., 2005). Por tanto, podríamos recomendar a los entrenadores que traten desarrollar la capacidad decisional de sus deportistas, durante el entrenamiento de la acción de saque. Así, en categorías de formación, es interesante el trabajo sobre saques tácticos, dirigidos a determinadas zonas del campo o jugadores, y el desarrollo de una óptima gestión del riesgo en el saque por parte del jugador, en función del momento y de la situación de juego.

\section{Agradecimientos}

Este estudio se ha realizado gracias a la aportación de la Consejería de Economía e Infraestructuras de la Junta de Extremadura a través del Fondo Europeo de Desarrollo Regional.

\section{Referencias}

Afonso, J., Esteves, F., Araújo, R., Thomas, L., \& Mesquita, I. (2012). Tactical determinants of setting zone in elite men's volleyball. Journal of Sports Science and Medicine, 11(1), 64-70.

Araújo, D., Davids, K., \& Hristovski, R. (2006). The ecological dynamics of decision making in sport. Psychology of sport and exercise, 7(6), 653-676.

Baker, F. C., Whiting, H. T. A., \& Van der Brugg, H. (1992). Prise de décisions dans les situacions sportives. En F. C. Baker, H. T. A. Whiting, y H. Van der Brugg (Eds.), Psychologie et Practiques Sportives. Concepts et Applications. France: Editions Vigot. Traducción española: Ediciones Morata. 
Bar-Eli, M., \& Raab, M. (2006). Judgment and decision making in sport an exercise: Rediscovery and new visions. Psychology of Sport and Exercise, 7, 519-524.

Coleman, J. E. (1975). A statiscal evaluation of selected volleyball techniques at the 1974 World's Volleyball Championships. Thesis Physical Education. Brigha Young University.

Conejero, M., Claver, F., Fernández-Echeverría, C., Gil-Arias, A., \& Moreno, M.P. (2017). Toma de decisiones y rendimiento en las acciones de juego intermedias y finalistas en voleibol, en sets con diferente resultado. Retos: nuevas tendencias en educación física, deporte y recreación, 31, 28-33.

Drikos, S., Kountouris, P., Laios, A. \& Laios, Y. (2009). Correlates of team performance in volleyball. International Journal of Performance Analysis in Sport, 9(2), 149-156.

Fernandes, S., \& Moutinho, C. (1996). A importância relativa da eficiência dos procedimentos de jogo na prestação competitiva de uma equipa de voleibol de rendimento. Estudos CEJD, 1, (72-77). Porto:FCDEF-UP.

Fernández-Echeverría, C., Claver, F., Gil, A., Del Villar, F., \& Moreno, M. P. (2015). Rol de juego y características del saque en voleibol infantil femenino. SPORT TK-Revista EuroAmericana de Ciencias del Deporte, 4(1), 49-56.

Fernandez-Echeverria, C., Gil, A., Moreno, A., Claver, F., \& Moreno, M. P. (2015). Analysis of the variables that predict serve efficacy in young volleyball players. International Journal of Performance Analysis in Sport, 15(1), 172-186.

Fleiss, J., Levin, B., \& Paik, M. (2003). Statistical methods for rates and proportions. NY: John Wiley \& Sons.

French, K. E. \& Thomas, J. R. (1987). The relation of knowledge development to children's basketball performance. Journal of Sport Psychology, 9, 15-32

García-González, L., Moreno, M.P., Moreno, A., Iglesias, D., \& Del Villar, F. (2009). Estudio de la relación entre conocimiento y toma de decisiones en jugadores de tenis, y su influencia en la pericia deportiva. Revista Internacional de Ciencias del Deporte, 17, 60-75.

Gréhaigne, J. F., Godbout, P. \& Bouthier, D. (2001). The teaching and learning of decision making in team sports. Quest, 53, 59-76.

Hastie, P. A., Sinelnikov, O. A. \& Guarino, A. J. (2009). The development of skill and tactical competencies during a season of badminton. European Journal Sport Science 66, 194201 istep:

Hepler, T. J., \& Chase, M. A (2008). Relationship between decision-making, task self-efficacy, and the performance of a sport skill. Journal of Sports Sciences, 26, 603-610.

Hodges, N. J., Huys, R., \& Starkes, J. L. (2007). Methodological review and evaluation of research in expert performance in sport. En Tenenbaum, G. Y Eklund, R. C. (Eds), Hanbook of Sport Psychology (161-183). New Jersey: John Wiley \& Sons.

Hodges, N. J., Starkes, J. L., \& MacMahon, C. (2006). Expert performance in sport: A cognitive process. In K. A. Ericsson, N. Charness, P. J. Feltovich, \& R. R. Hoffman (Eds.). The Cambridge handbook of expertise and expert performance (471-488). Cambridge, New York: Cambridge University Press.

Iglesias, D., Moreno, M. P., Santos-Rosa, F. J., Cervelló, E. M. \& Del Villar, F. (2005). Cognitive expertise in sport: relationships between procedural knowledge, experience and performance in youth basketball. Journal Human Movement Study 49, 65-76. 
Iglesias, D., Sanz, D., García-Calvo, T., Cervelló, E. M. \& Del Villar, F. (2005). Influencia de un programa de supervisión reflexiva sobre la toma de decisiones y la ejecución del pase en jóvenes jugadores de baloncesto. Revista de Psicología del Deporte, 14(2), 209-223.

Janelle, C. M. \& Hillman, C. H. (2003). Expert performance in sport: Current perspectives and critical issues. En J. L. Starkes y K. A. Ericsson (Eds.), Expert performance in sports: Advances in research on sport psychology (19-47). Champaign, IL: Human Kinetics.

Johnson, J. G. (2006). Cognitive modeling of decision making in sports. Psychology of Sport and Exercise, 7, 631-652.

Köppen, J. \& Raab, M. (2009). Knowledge of athletes as cues for simple choices. En D. Araújo, H. Ripoll y M. Raab (Eds.), Perspectives on Cognition and Action in Sport (pp. 108-117). Nueva York: Nova Science Publishers.

McPherson, S. L. (1993a). The influence of player experience on problem solving during batting preparation in baseball. Journal of Sport and Exercise Psychology, 15, 304-325.

McPherson, S. L. (1993b). Knowlegde representation and decision-making in sport. En J. L. Starkes y F. Allard (Eds.), Cognitive issues in motor expertise (159- 188). Amsterdam: Elsevier.

McPherson, S. L. (1999a). Expert-novice differences in performance skills and problem representations of youth and adults during tennis competition. Journal Exercise and Sport 70, 233-251.

Monge, M. A. (2007). Construcción de un sistema observacional para el análisis de la acción de juego en voleibol. A Coruña: Servicio de Publicaciones.

Moran, A. P. (2012). Thinking in action: Some insights from cognitive sport psychology. Thinking Skills and Creativity, 7, 85-92.

Moreno, A., Del Villar, F., García-González, L., Gil, A., \& Moreno, M. P. (2011). Intervención en la toma de decisiones en jugadores de voleibol en etapas de formación. Revista de Psicología del Deporte, 20(2), 785-800.

Moreno, A., Moreno, M. P., Iglesias, D., García-González, L. \& Del Villar, F. (2006). Estudio del conocimiento declarativo en función de la experiencia y de la edad en jugadores jóvenes de voleibol. Cultura, Ciencia y Deporte, 5(2), 73- 80.

Moreno, M. P., Moreno, A., García-González, L., Gil, A., Claver, F., \& Del Villar, F. (2011). Elaboración de herramientas cognitivas para la detección, seguimiento y optimización de talentos deportivos en voleibol. Arvhivos de Medicina del Deporte, 146(28), 435-446.

Murray, M. (1991). Development of decision and execution components of blocking performance in volleyball. Tesis doctoral. Universidad de Oklahoma.

Nielsen, T. M. \& McPherson, S. L. (2001). Response selection and execution skills of professionals and novices during singles tennis competition. Percept Motor Skills, 93, 541-555.

Oslin, J. L., Mitchell, S. A., \& Griffin, L. L. (1998). The Game Performance Assessment Instrument (GPAI): Development and Preliminary Validation. Journal of Teaching in Physical Education, 17(2), 231-243.

Palao, J.M., Santos, J. \& Ureña, A. (2004). Efecto del tipo y eficacia del saque sobre el bloqueo y el rendimiento del equipo en defensa. RendimientoDeportivo.Com,8. $<\mathrm{http} / / / \mathrm{www}$.rendimientodeportivo.com/N008/Artic040.htm>. 
Papadimitriou, K., Pashali, E., Sermaki, I., Mellas, S. \& Papas, M. (2004). The effect of the opponents' serve on the offensive actions of Greek setters in volleyball games. International Journal of Performance Analysis in Sport, 4(1), 23-33.

Rodriguez-Ruiz, D., Quiroga, M. E., Miralles, J. A., Sarmiento, S., de Saá, Y., \& García-Manso, J. M. (2011). Study of the technical and tactical variables determining set win or loss in toplevel European men's volleyball. Journal of Quantitative Analysis in Sports, 7(1), 1-15.

RFEVB. Reglas Oficiales de Voleibol. 2013-2016. RFEVB, Madrid. SEEP]

Ruiz, L. M., Sánchez, M., \& Piqueras, J. D. (2006). Los expertos en el deporte: Su estudio y análisis desde una perspectiva psicológica. Anales de psicología, 22(1), 132.

Sellinger, A. \& Ackermann-Blount, J. (1985). El Voleibol de Potencia. Buenos Aires. Confederación Argentina de Voleibol.

Starkes, J. L. (1993). Motor experts: Opening thoughts. En J. L. Starkes y F.Allard (Eds.), Cognitive issues in motor expertise (pp. 3-16). Amsterdam: Elsevier.

Starkes, J. L., Helsen, W., \& Jack, R. (2001). Expert performance in sport and dance (pp. 174201). En R. N. Singer, H. A. Hausenblas y C. M. Janelle (Eds.). Handbook of sport psychology (3rd edition). New York: John Wiley \& Sons.

Sternberg, S. (1969). The discovery of processing stages. Acta Psychology, 30.

Sutton, J., \& Mcilwain, D. J. F. (2015). Breadth and Depth of Knowledge in Expert versus Novice Athletes. In D. Farrow \& Baker (Eds.), The Routledge Handbook of Sports Expertise (pp. 95-105). New York: Routledge.

Tabachnick, B. G., \& Fidell, L. S. (2007). Using multivariate statistics. Boston: Allyn and Bacon.

Tenembaum, G. (2004). Decision Making in sport. En C. Spielber (Ed.) Encyclopedia of Applied Psychology, Volume 1. (pp. 575-584). Amsterdam: Elsevier Academic Press.

Thomas, K. T. \& Thomas, J. R. (1994). Developing expertise in sport: The relation of knowledge and performance. International Journal of Sport Psychology, 25, 295-315.

Ureña, A. \& González, M. (2006). Manual del preparador de voleibol Nivel II. Cádiz: Federación Andaluza de Voleibol.

Ureña, A., Calvo., R.M. \& Lozano, C. (2002). Estudio de la recepción del saque en el voleibol masculino español de elite tras la incorporación del jugador libero. Revista internacional de medicina y ciencias de las actividad física y del deporte, 2(4), 37-49.

Ureña, A., Santos, J. A., Martínez, M., Calvo, R., \& Oña, A. (2010). La facilitación defensiva a través del saque en el voleibol femenino de alto nivel. European Journal of Human Movement, (6), 175-189.

Ureña, A., Vavassori, R., León, J., \& González, M. (2011). Efecto del saque en suspensión sobre la construcción del ataque en el voleibol.(jump serve incidence on the attack phase in the spanish under-14 volleyball). RICYDE.Revista Internacional De Ciencias Del Deporte.Doi: 10.5232/ricyde, 7(26), 384-392.

Van Orden, G. C., Holden, J. G., \& Turvey, M. T. (2003). Self-organization of cognitive performance. Journal of Experimental Psychology: General, 132(3), 331.

Williams, A. M., Davids, K., \& Williams, J. G. (1999). Visual perception and action in sport. London: E \& FN Spon.

Williams, A. M., Ward, P., Smeeton, N. J. \& Allen, D. (2004). Developing anticipation skills in tennis using on-court instruction: perception versus perception and action. Journal of Applied Sport Psychology, 16(4), 350-360. 